\title{
Selectivity of Multi-wall Carbon Nanotube Network Sensoric Units to Ethanol Vapors Achieved by Carbon Nanotube Oxidation
}

\author{
Robert Olejnik \\ Polymer Centre, Faculty of Technology, T. Bata University, T.G.M. 275, 76272 Zlin, Czech Republic \\ $\&$ \\ Centre of Polymer Systems, University Institute, T. Bata University \\ Nad Ovcirnou 3685, 76001 Zlin, Czech Republic \\ E-mail: rolejnik@volny.cz \\ Petr Slobodian (Corresponding author) \\ Polymer Centre, Faculty of Technology, T. Bata University, T.G.M. 275, 76272 Zlin, Czech Republic \\ $\&$ \\ Centre of Polymer Systems, University Institute, T. Bata University \\ Nad Ovcirnou 3685, 76001 Zlin, Czech Republic \\ Tel: $420576031350 \quad$ E-mail: slobodian@ft.utb.cz \\ Pavel Riha \\ Institute of Hydrodynamics, Academy of Sciences, Pod Patankou 16612 Prague, Czech Republic \\ E-mail: riha@ih.cas.cz \\ Petr Saha \\ Polymer Centre, Faculty of Technology, T. Bata University, T.G.M. 275, 76272 Zlin, Czech Republic
}

\&

Centre of Polymer Systems, University Institute, T. Bata University

Nad Ovcirnou 3685, 76001 Zlin, Czech Republic

E-mail: saha@utb.cz

Received: October 8, 2011 Accepted: October 27, $2011 \quad$ Published: January 1, 2012

doi:10.5539/jmsr.v1n1p101 URL: http://dx.doi.org/10.5539/jmsr.v1n1p101

This project was supported by the internal grant of TBU in Zlin No. IGA/3/FT/11/D funded from the resources of specific University research, the Operational Program Research and Development for Innovations co-funded by the European Regional Development Fund (ERDF) and national budget of the Czech Republic within the framework of the Centre of Polymer Systems project (reg.number: CZ.1.05/2.1.00/03.0111), the Fund of the Institute of Hydrodynamics AVOZ20600510 and the Czech Ministry of Education, Youth and Sports project (MSM 7088352101).

\footnotetext{
Abstract

Two kinds of multiwall carbon nanotubes (MWCNT) networks "Buckypaper" were made by the vacuum filtration method of MWCNT aqueous suspension. The first one was prepared from pure CNT and the second from its oxidized form. The CNT oxidation increase content of oxygen bonded to the surface of CNT. The sensitivity of MWCNT networks to organic solvent vapors (ethanol and heptane) has been investigated by
} 
resistance measurements. The solvents had different polarities given by Hansen solubility parameters and nearly the same volume fractions of saturated vapors at the condition of experiment. CNT oxidation significantly increases the sensitivity of CNT resistive sensoric unit to vapors of ethanol and decrease response to heptane vapors.

Keywords: Carbon nanotube network, Buckypaper, Oxidation, Sensor, Electrical resistance

\section{Introduction}

Carbon nanotubes (CNTs) have raised much interest during the recent years due to their inherent extraordinary electrical and mechanical properties. Moreover CNTs show remarkable sensitivity to the change of chemical composition of the surrounding environment. Gas and vapor adsorption as well as desorption usually proceeds at high rates and amounts (Hussain et al., 2008). This property is favorable for their use in the form of membranes (Smajda et al., 2007), adsorbents (Agnihotri et al., 2006) or gas sensors (Romanenko et al., 2007; Qureshi et al., 2009; Brahim et al., 2009; Ouyang and Li, 2010).

The molecules are adsorbed on CNT surface by van der Waals attracting forces, which leads to remarkable changes in CNT electrical resistance. Physisorbed molecules influence the electrical properties of isolated CNT and also of inter-tube contacts (Mowbray et al., 2009; Slobodian et al., 2011). The resistance of macroscopic CNT objects like aggregates or network structures used in gas sensors is predominantly determined by contact resistance of crossing tubes, rather than by resistance of CNT segments. Here, the tubes are much shorter than sensor dimensions and inter-tube contacts act as parallel resistors between highly conductive CNT segments. It was found that so called "Buckypaper", network prepared from entangled CNTs by vacuum filtration is capable to detect organic vapors in air (Kastanis et al., 2007).

Carbon nanotutubes can be used as resistive gas sensors for detection of volatile organics compounds (VOC). Several principles of sensing mechanisms can be employed. It goes from the change of intrinsic resistance of individual tubes by exposure to reducing methanol vapors ( $\mathrm{Li}$ et al., 2007) (here fixed in PMMA matrixes) or in the form of thin polymeric film with percolating CNT network (Philip et al., 2003; Greenshields et al., 2011) or using of electrically conductive network structures made of carbon nanotubes with substantial role of inter-tube contacts (similar to present contend). Such carbon nanotube networks ("Buckypaper") can be used for detection by variation of ways. It can be made from pure tubes (Kastanis et al., 2007) or their wet chemical oxidized forms (Slobodian et al.) enhancing selectivity to oxygenated kinds of VOC or by dry functionalization by oxygen or fluorine plasma (Liu et al., 2007) increasing response to ethanol. Another variation of this principle can be found in the literature like printing of CNT layer onto flexible PET foil (Parikh et al., 2007), conductive PMMA/CNT composite prepared as spray layered of PMMA microbeads decorated by CNT (Feller et al., 2011) next one can be mentioned as increasing of sensitivity to vapors with affine polarity by CNT covalent functionalization by PMMA (Olejnik et al., 2011) or CNT network made of adsorbed carbon nanotubes onto skeleton made of electrospuned nylon 6,6 nanofibers applied as sensor for low molecular weight alcohols (Choi et al., 2010).

The quality of aqueous dispersion of nanotubes, the porosity of formed networks as well as nanotube adherence, intercontact resistance, etc., can be suitably influenced by a nanotube surface functionalization such as oxidation (Kastanis et al., 2007). It was found that the oxidized CNTs form a network with more uniform pore structure and dense morphology with lower porosity in comparison with networks formed by pristine nanotubes. The network structure results from fine nanotube aqueous dispersion, and thus deposition of individual CNT and/or only small CNT agglomerates on filtrating membrane.

CNT oxidation in $\mathrm{KMnO}_{4} / \mathrm{H}_{2} \mathrm{SO}_{4}$ mixture causes nanotube shortening, creation of defect sites and open ends (Rasheed et al., 2007; Hernadi, et al., 2001). Potassium permanganate $\left(\mathrm{KMnO}_{4}\right)$ produce carboxylic acid groups (-COOH) on CNT surface as well as a significant amount of other oxygenated functional groups such as hydroxyl $(-\mathrm{OH})$ and carbonyl $(=\mathrm{O})$ groups (Rasheed et al., 2007; Hernadi, et al., 2001). Small amount of amorphous carbon after the $\mathrm{KMnO}_{4}$ oxidation process can be also expected (Hernadi, et al., 2001). Nevertheless, the other study shows that oxidation by $\mathrm{KMnO}_{4}$ in an acidic suspension provides treated nanotubes free of amorphous carbon (Rasheed et al., 2007).

Even if carbon nanotubes are presently used for detection of gases the sensors are quite expensive and difficult to produce. Simpler and cheaper ways to detect gases can be reflected in practical application of CNT network structure. The present work describes increased sensitivity and achieved selectivity to vapors of ethanol by CNT oxidation in $\mathrm{KMnO}_{4} / \mathrm{H}_{2} \mathrm{SO}_{4}$ solution. 


\section{Experimental}

\subsection{Materials}

Multiwall carbon nanotubes (MWCNTs) Baytubes C150, produced by chemical vapor deposition were supplied by Bayer Material Science AG, Germany (diameter 5-20 nm, length 1-10 $\mu \mathrm{m}, \mathrm{C}$-purity $>99 \%$ and bulk density $140-230 \mathrm{~kg} / \mathrm{m}^{3}$ declared by supplier). The oxidized material was prepared by following procedure: glass reactor, $300 \mathrm{~cm}^{3}$ of $0.5 \mathrm{M} \mathrm{H}_{2} \mathrm{SO}_{4}, 1.5 \mathrm{~g}$ of $\mathrm{KMnO}_{4}$ and $0.5 \mathrm{~g}$ of MWCNTs, the dispersion was sonicated at $40^{\circ} \mathrm{C}$ using UP-400S Dr. Hielsher GmbH Apparatus (ultrasonic horn H7, amplitude $\mu \mathrm{m}$, power density $300 \mathrm{~W} / \mathrm{cm}^{3}$, frequency $24 \mathrm{kHz}$ ) for one hour at $50 \%$ power of the apparatus and in $50 \%$ pulse mode, the product was filtered and washed with concentrated $\mathrm{HCl}$ to remove $\mathrm{MnO}_{2}$.

MWCNT networks (Buckypaper) (MWCNT-N), were prepared by CNT dispersion vacuum filtration thought polyurethane membrane prepared by technology of electrospinning (Kimmer et al., 2009). Aqueous paste was prepared using a mortar and pestle. The paste was diluted in DI water with sodium dodecyl sulfate (SDS). The final nanotube concentration in the dispersion was $0.03 \mathrm{wt} . \%$. The dispersion was sonicated in Dr. Hielscher $\mathrm{GmbH}$ apparatus (ultrasonic horn S7, amplitude $88 \mu \mathrm{m}$, power density $300 \mathrm{~W} / \mathrm{cm}^{2}$, frequency $24 \mathrm{kHz}$ ) for 2 hours and the temperature of ca $50^{\circ} \mathrm{C}$. The final MWCNT dispersion was centrifuged in Hettich-Zentrifugen (Rotina 35) at $4000 \mathrm{rpm}$ for $15 \mathrm{~min}$. Supernatant was used for filtration. The formed disk-shaped networks were washed several times by deionized water and methanol in situ, then peel off the filtering membrane and dried between filter papers at RT.

\subsection{Instruments}

The structure of MWCNT networks was observed by means of scanning electron microscope (SEM) Vega LMU, produced by Tescan Ltd. The samples were deposited on carbon targets and covered with a thin Au/Pd layer. For the observations the regime of secondary electrons was chosen. The content of oxygen in both forms of MWCNT networks was detected with help of X-ray spectroscopy (EDX) which was in accessories of microscope.

\subsection{Reactions}

The stripes made of CNT networks (length $15 \mathrm{~mm}$, width $5 \mathrm{~mm}$, thickness ca $0.15 \mathrm{~mm}$ ) were exposed to the vapors of two different solvents, namely ethanol and heptane. The electrical resistance of stripes was measured along the specimen length by the two-point technique using multimeter Sefram 7338. The stripe was placed on a planar holder with $\mathrm{Cu}$ electrodes fixed on both sides of the stripe. Time-dependent electrical resistance measurements were performed during adsorption and desorption cycles. The holder with the specimen was quickly transferred into an airtight conical flask full of vapors of the respective solvent a layer of which was at the bottom. The measurements were conducted in saturated vapors at atmospheric pressure, temperature $25^{\circ} \mathrm{C}$ and relative humidity $60 \%$. After 6 minutes of measurement the holder was promptly removed from the flask and for the next 6 minutes the sample resistance was measured in the mode of desorption. Four different specimens were measured for each MWCNT network and solvent.

\section{Results}

SEM analyses of upper surfaces of different kind of papers prepared in the course of optimalization process to achieve the smoothest "Buckypaper" surface are presented in Fig. 1. In all cases it demonstrates CNT network as a porous structure created from entangled nanotubes with inter-tube pores. Fig. 1A shows a network fabricated from CNT dispersion prepared only by sonication, B) shows a network made after milling CNT agglomerates before sonication and finally $\mathrm{C}$ ) shows a network when dispersion of milled and sonicated CNTs was centrifuged. Fig. 2D represents the upper surface of CNT network made from $\mathrm{KMnO}_{4}$ oxidized tubes. The original MWCNT material is supplied in form of CNT granules. It seems that MWCNTs are not enough individualized from granules by sonication only, since MWCNT network surface is wrinkled by remaining MWCNT granules, Fig.1A. The wrinkles are partially eliminated by MWCNT milling using a mortar and pestle before sonication since MWCNT granules nearly disappears, Fig. 2B. However, there are probably still some MWCNT aggregated to submicron aggregates which can be eliminated by centrifugation, Fig. 1C. This MWCNT network is the most uniform, smooth and crack-free. Finally, the surface of the MWCNT network made of oxidized tubes seems to be smoother than all made of pure MWCNT with more densely packed tubes and a smaller diameter of inter-tube pores. Moreover, EDX measurements proved an increase of oxygen content on the surface of MWCNT from 4.86 at. $\%$ on pure MWCNT network to $21.26 \%$ on network made from $\mathrm{KMnO}_{4}$ oxidized tubes.

The strips made of optimized MWCNT network (Fig. 1C) and the network of $\mathrm{KMnO}_{4}$ oxidized tubes were exposed to the vapor of two different solvents. The solvents were chosen to have similar values of saturated 
vapor pressure, $p_{i}$, which defines corresponding volume fractions of vapors in air, $x_{i}$, with considerably different polarities defined by Hildebrand solubility parameter, $\delta_{t}$. As good candidates to satisfy these criteria the organics solvents heptane and ethanol were selected. Calculated values of $x_{i}$ are 7.8 vol. $\%$ for ethanol and 6.0 vol. \% for heptane at $25^{\circ} \mathrm{C}$ with $\delta_{t}=15 \mathrm{Mpa}^{1 / 2}$ and $\delta_{t}=26 \mathrm{Mpa}^{1 / 2}$ for heptane and ethanol, respectively.

The typical adsorption/desorption behavior of MWCNT networks exposed to/disposed from heptane and ethanol during two cycles are presented in Fig. 2. The experimental curves represents measured data for a pure MWCNT network, part a), and the network made of oxidized tubes, part b), as an average values (4 specimens) with standard deviations represented by error bars. Over all, the adsorption of organics molecules increase resistance with time, which is presented in the figure as sensitivity, $S$, defined by Eq. (1),

$$
S=\frac{R_{g}-R_{a}}{R_{a}}=\frac{\Delta R}{R_{a}}
$$

Here $R_{a}$ represents specimen resistance in air and $R_{g}$ resistance of the specimen exposed to vapor, $\Delta R$ stands for the resistance change. There is observed an initial sharp increase in $S$ followed by a slower phase. Simultaneously, the organic molecules are removed in the course of desorption and the specimen resistance recovers the initial value. This desorption part starts again by a rapid sensitivity decrease followed by a slower decrease to a constant value within time of the measurement. The mechanism of macroscopic resistance change can be explained by formation of non-conducting layers between nanotubes (Romanenko et al., 2007). It lowers the quality of inter-tube contacts and also their number leading directly to change in macroscopic resistance of MWCNT strip. In any case, the studied macroscopic resistance response was found to be reversible and reproducible.

It can be recapped on basis of the measured data that used MWCNT networks are sensitive to the change of its surrounding for system like air/organics solvent. The measured sensitivities after 6 min of exposition are presented in Table 1. The network made of pure MWCNT seems to have the same sensitivity for heptane and ethanol when experimental errors are taken into account. On the contrary, the sensitivity values and their ratio, $\mathrm{S}_{\text {oxidised }} / \mathrm{S}_{\text {pure }}$, for oxidized form of MWCNT network demonstrates significant increase of sensitivity to polar ethanol compared to the sensitivity decrease for non-polar heptane. This is probably caused by the increased polarity of oxidized tubes and better affinity of ethanol molecules to the MWCNT surface.

\section{Conclusions}

Multiwall carbon nanotubes were used in their pure and oxidized form to prepare entangled networks (buckypaper) whose response to two organic solvent vapors was measured by their electrical resistance. The results show that the prepared networks are capable to detect vapors in the air. The MWCNT network can be considered as a suitable material for application as a cheap and easy to prepare micro-sized vapor sensing element, which is sensitive, selective and has reversible and reproducible properties. The sensor sensitivity to ethanol vapors was effectively improved by MWCNT oxidation by acidic $\mathrm{KMnO}_{4}$.

\section{References}

Agnihotri, S., Mota, J. P. B., Rostam-Abadi, M., \& Rood, M. J. (2006). Theoretical and experimental investigation of morphology and temperature effects on adsorption of organic vapors in single-walled carbon nanotubes. Journal of Physical Chemistry B, 110(15), 7640-7647. http://dx.doi.org/10.1021/jp060040a

Brahim, S., Colbern, S., Gump, R., Moser, A., \& Grigorian, L. (2009). Carbon nanotube-based ethanol sensors. Nanotechnology, 20, 235502. http://dx.doi.org/10.1088/0957-4484/20/23/235502

Hernadi, K., Siska, A., Thien-Nga, L., Forro, L., \& Kiricsi, I. (2001). Reactivity of different kinds of carbon during oxidative purification of catalytically prepared carbon nanotubes. Solid State Ionics, 141(1), 203-209. http://dx.doi.org/10.1016/S0167-2738(01)00789-5

Hussain, C.-M., Saridara, C., \& Mitra, S. (2008). Microtrapping characteristics of single and multi-walled carbon nanotubes. Journal of Chromatography A, 1185(2), 161-166. http://dx.doi.org/10.1016/j.chroma.2008.01.073

Kastanis, D., Tasis, D., Papagelis, K., Parthertios, J., Tsakiroglou, C., \& Galiotis, C. (2007). Oxidized multi-walled carbon nanotube film fabrication and characterization. Advanced Composites Letters, 16(6), 243-248.

Kimmer, D., Slobodian, P., Petras, D., Zatloukal, M., Olejnik, R., \& Saha, P. (2009). Polyurethane/MWCNT nanowebs prepared by electrospinning process. Journal of Applied Polymer Science, 111(6), 2711-2714. http://dx.doi.org/10.1002/app.29238 
Mowbray, D. J., Morgan, C., \& Thygesen, K. S. (2009). Influence of $\mathrm{O}_{2}$ and $\mathrm{N}_{2}$ on the conductivity of carbon nanotube networks. Physical Review B, 79(19), Article Number 195431. http://link.aps.org/doi/10.1103/PhysRevB.79.195431

Ouyang, M., \& Li, W. J. (2010). Performance of F-CNTs sensors towards ethanol vapor using different functional groups. Proceedings of the $5^{\text {th }}$ IEEE International Conference on Nano/Micro Engineered and Molecular Systems, January 20-23, Xiamen, China. http://dx.doi.org/10.1109/NMDC.2010.5651960

Qureshi, A., Kang, W. P., Davidson, J. L., \& Gurbuz, Y. (2009). Review on carbon-derived, solid-state, micro and nano sensors for electrochemical sensing application, Diamond and Related Materials, 18(12), 1401-1420. http://dx.doi.org/10.1016/j.diamond.2009.09.008

Rasheed, A., Howe, J. Y., Dadmun, M. D., \& Britt, P. F. (2007). The efficiency of the oxidation of carbon

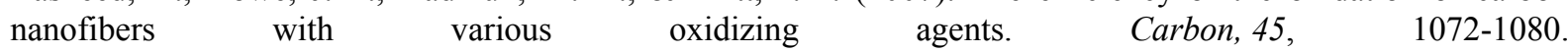
http://dx.doi.org/10.1016/j.carbon.2006.12.010

Romanenko, A. I., Anikeeva, O. B., Kuznetsov, V. L., Buryakov, T. I., Tkachev, E. N., \& Usoltseva, A. N. (2007). Influence of helium, hydrogen, oxygen, air and methane on conductivity of multiwalled carbon nanotubes. Sensors and Actuators A, 138(2), 350-354. http://dx.doi.org/10.1016/j.sna.2007.05.010

Smajda, R., Kukovecz, A., Konya, Z., \& Kiricsi, I. (2007). Structure and gas permeability of multi-wall carbon nanotube buckypapers. Carbon, 45(6), 1176-1184. http://dx.doi.org/10.1016/j.carbon.2007.02.022

Slobodian, P., Riha, P., Lengalova, A., Svoboda, P., \& Saha, P. (2011). Multi-wall carbon nanotube networks as potential resistive gas sensors for organic vapor detection. Carbon, 49(7), 2499-2507. http://dx.doi.org/10.1016/j.carbon.2011.02.020

Liu, C. K., Wu, J. M., \& Shih, H. C. (2010). Application of plasma modified multi-wall carbon nanotubes to ethanol vapor detection. Sensors and Actuators B-Chemical, 150(2), 641-648. http://dx.doi.org/10.1016/j.snb.2010.08.026

Greenshields, MWCC, Meruvia, MS, Hummelgent, IA, Coville, NJ, Mhlanga, SD, Ceragioli, HJ, Quispe, JCR, \& Baranauskas, V. (2011). AC-Conductance and Capacitance Measurements for Ethanol Vapor Detection Using Carbon Nanotube-Polyvinyl Alcohol Composite Based Devices. Journal of Nanoscience and Nanotechnology, 11(3), 2384-2388. http://dx.doi.org/10.1166/jnn.2011.3518

Choi, J, Park, EJ, Park, DW, \& Shim, SE. (2010). MWCNT-OH adsorbed electrospun nylon 6,6 nanofibers chemiresistor and their application in low molecular weight alcohol vapours sensing. Synthetic Metals, 160(23-24), 2664-2669. http://dx.doi.org/10.1016/j.synthmet.2010.10.022

Shih, Y. H., \& Li, M. S. (2007). Adsorption of selected volatile organic vapors on multiwall carbon nanotubes Journal of Hazardous Materials, 154(1-3), 21-28. http://dx.doi.org/10.1016/j.jhazmat.2007.09.095

Parikh, K, Cattanach, K, Rao, R, Suh, DS, Wu, AM, \& Manohar, SK. (2005). Flexible vapour sensors using single walled carbon nanotubes, Sensors and Actuators B-Chemical, 113(1), 55-63. http://dx.doi.org/10.1016/j.snb.2005.02.021

Philip, B, Abraham, JK, Chandrasekhar, A, \& Varadan, VK. (2003). Carbon nanotube/PMMA composite thin films for gas-sensing applications. Smart Materials \& Structures, 12(6), 935-939, Article Number: PII S0964-1726(03)68091-2. http://dx.doi.org/10.1088/0964-1726/12/6/010

Olejnik R., Slobodian P., Riha P., \& Machovsky M. Increased sensitivity of multi-walled carbon nanotube network by PMMA functionalization to vapors with affine polarity. Journal of Applied Polymer Science, paper in press.

Feller, J. F., Lu, J., Zhang, K., Kumar, B., Castro, M., Gatt, N., \& Choi, H. J. (2011). Novel architecture of carbon nanotube decorated poly(ethyl methacrylate) microbead vapour sensors assembled by spray layer by layer. J Mater Chem, 21(12), 4142-4149. http://dx.doi.org/10.1039/c0jm03779f 
Table 1. Increase of sensitivity of pure MWCNT network and the network made from oxidized tubes exposed to saturated vapors of heptane and ethanol

\begin{tabular}{|c|c|c|c|}
\hline Organic solvent & $S_{\text {pure }}[\%]$ & $S_{\text {oxidised }}[\%]$ & $\mathrm{S}_{\text {oxidised }} / \mathrm{S}_{\text {pure }}$ \\
\hline heptane & $11.7 \pm 0.9$ & $7.4 \pm 1.3$ & 0.63 \\
\hline ethanol & $11.3 \pm 0.8$ & $34.0 \pm 2.0$ & 3.0 \\
\hline
\end{tabular}

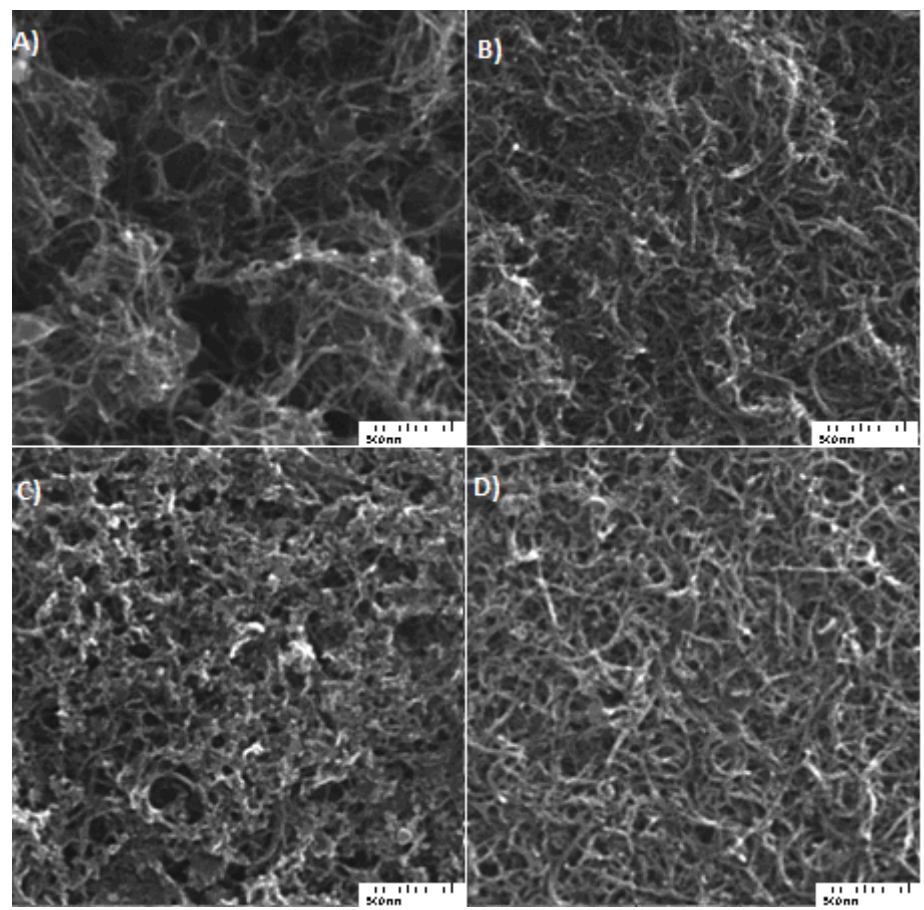

Figure 1. SEM analyses of upper surfaces of MWCNT networks. A) The network prepared from MWCNT dispersion treated by sonication only. B) The network prepared from milled and sonication treated MWCNT. C) The network prepared from dispersion of milled, sonicated MWCNT and centrifuged MWCNT. D) MWCNT network made from $\mathrm{KMnO}_{4}$ oxidized tubes

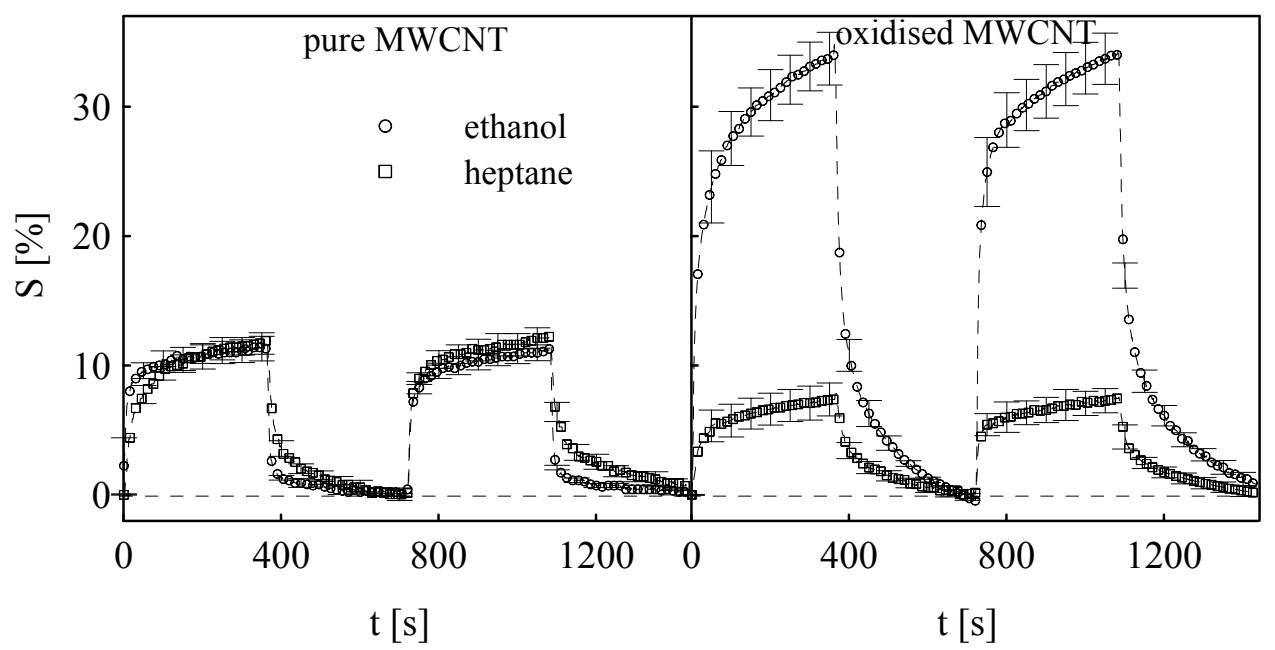

Figure 2. Two adsorption/desorption cycles for pure MWCNT and oxidized MWCNT networks exposed to vapors of heptane (filled circles) and ethanol (open circles) 